\title{
Assessment of the anti-obesity effects of the TNP-470 analog, CKD-732
}

\author{
Yoo Mee Kim, Juan Ji An ${ }^{1,2}$, Yong-Jun Jin ${ }^{4}$, Yumie Rhee ${ }^{1,2,3}$, Bong Soo Cha ${ }^{1,2,3}$, \\ Hyun Chul Lee $\mathrm{C}^{1,2,3}$ and Sung-Kil Lim ${ }^{1,2,3}$
}

Division of Endocrinology, Department of Internal Medicine, NHIC Ilsan Hospital, Goyang, Republic of Korea

${ }^{1}$ Division of Endocrinology, Department of Internal Medicine, Yonsei University, College of Medicine, Seoul, South Korea

${ }^{2}$ BK21 Project for Medical Science, College of Medicine, Yonsei University, Seoul, South Korea

${ }^{3}$ College of Medicine, Institute of Endocrine Research, Yonsei University, Seoul, South Korea

${ }^{4}$ Division of Endocrinology, Department of Internal Medicine, YanBian University, Medical College, Affiliated Hospital, YanJi, People's Republic of China

(Requests for offprints should be addressed to S-K Lim who is now at Division of Endocrinology, Department of Internal Medicine, College of Medicine, Yonsei University, 134 Shinchon-dong Seodaemun-ku, PO Box 120-749, Seoul, South Korea; Email: Isk@yumc.yonsei.ac.kr)

\begin{abstract}
The systemic treatment with angiogenesis inhibitor has been shown to result in weight reduction and adipose tissue loss in various models of obesity. To verify the mechanism of CKD-732 (TNP-470 analog) against obesity, we evaluated CKD-732's peripheral and central anti-obesity effects. CKD-732 was injected subcutaneously (s.c.) for 7 days in various animal models and intracerebroventricularly (i.c.v.) in arcuate nucleus (ARC) lesion mice, ob/ob mice, and normal littermates. Modulation of the hypothalamic neuropeptide mRNAs after i.c.v. injection was evaluated in ARC lesion mice and normal littermates. A conditioned taste aversion (CTA) was performed using lithium chloride (LiCl) as a positive control agent in Long-Evans Tokushima Otsuka and Otsuka Long-Evans Tokushima fatty (OLETF) rats. As a result, 7 days of CKD-732 s.c. injection reduced the cumulative food intake and the body weight significantly in both treated obese (e.g. $114 \cdot 8 \pm 13.4 \mathrm{~g}$ vs $170 \cdot 7 \pm 20 \cdot 6 \mathrm{~g}, 7 \cdot 9 \pm 0 \cdot 5 \%$ decrease vs $0 \cdot 3 \pm 2 \cdot 2 \%$ decrease; in treated OLETF rat versus control OLETF rat, $P<0.01$ respectively) and non-obese models. Epididymal and mesenteric fat pads, and the size of adipocytes were significantly decreased in treated rats. A single i.c.v. injection decreased food intake and body weight in $A R C$ lesion mice and $o b / o b$ mice but not in normal littermates. Unexpectedly, the hypothalamic neuropeptide mRNAs were not altered by single i.c.v. injection. CKD-732 also induced a dose-dependent CTA comparable with LiCl injection, which is a commonly used agent to produce a CTA. In conclusion, CKD-732 causes significant body weight and appetite reduction, possibly by decreasing adiposity directly and inducing central anorexia, which is partly explained by a CTA. These results should be carefully verified to assess the utility of CKD-732 as an anti-obesity drug.
\end{abstract}

Journal of Molecular Endocrinology (2007) 38, 455-465

\section{Introduction}

Obesity is highly prevalent in many developed countries and its prevalence is increasing worldwide (Kopelman 2000). Diet and exercise remain the cornerstones of obesity management; however, it is likely that many patients will require an anti-obesity drug to reduce body weight and prevent complications (Bray \& Tartaglia 2000). As drugs used in the past to treat obesity have caused unacceptable mortality and morbidity, there is a need for more effective and better tolerated anti-obesity drugs (Weigle 2003). Impaired fuel metabolism is an early pathogenic factor in obesity and type 2 diabetes mellitus (Minokoshi et al. 2002). Frequently, daily energy intake is variable within and among individuals, and is not well correlated with daily energy expenditure. Despite short-term mismatches in energy balance, however, most people match cumulative energy intake and expenditure with great precision over a period that spans many meals. This phenomenon reflects an active regulatory process, termed energy homeostasis, which promotes stability in the amount of body energy stored in the form of fat (Schwartz et al. 2000). Among these regulatory processes, the central regulation of fuel homeostasis has been highlighted.

In searching for new anti-obesity drugs, Rupnick $e t$ al. (2002) have recently speculated that neovascularization may be critical for adipose tissue growth. They showed that systemic treatment with angiogenesis inhibitors resulted in weight reduction and adipose tissue loss in various mouse models of obesity (Rupnick et al. 2002). This concept was developed due to the unique feature of the microvasculature within adipose tissue. Since adipose tissue has enormous growth potential given the appropriate metabolic challenge, it displays a high degree of plasticity with respect to its vascularization. 
Interestingly, angiogenesis, or the formation of new blood vessels from existing ones, often precedes adipogenesis in developing adipose tissue microvasculature (Crandall et al. 1997).

The concept linking new blood vessel formation with tumor growth and metastases was proposed more than 20 years ago. Since Ingber et al. (1990) discovered that fumagillin from Aspergillus fumigatus inhibits new blood vessel growth, many semi-synthetic fumagillin analogs have been synthesized from fumagillol, the hydrolysis product of fumagillin. Of these angiostatic compounds, fumagillin and its derivative, TNP-470, inhibit neovascularization via endothelial cell cycle arrest in the late G1 phase (Abe et al. 1994). Methionine aminopeptidase-2 (MetAP2) has been identified as a molecular target. However, the link between MetAP2 inhibition and endothelial cell cycle arrest by fumagillin and TNP-470 remains unclear (Griffith et al. 1997, Sin et al. 1997). We used a synthetic derivative of TNP470 , which has been proven to have higher affinity to MetAP2 and higher efficacy on suppression of in vivo tumor growth compared with TNP-470 (Chun et al. 2005). Previous reports have suggested that TNP-470 reduces body weight and slightly decreases food intake in various animal models of obesity (Rupnick et al. 2002). However, the precise mechanism of action remains unknown, and the long-term safety of the drug aimed at anti-obesity treatment has not been clearly proven.

Among various endeavors to develop new drugs for obesity, many data indicate that pharmacotherapies based on the melanocortin (MC) system might provide useful tools in the treatment of obesity. This neuropeptide system is now thought to be critical for the regulation of food intake and energy balance (Fan et al. 1997, Schwartz et al. 1997, Thornton et al. 1997, Mizuno et al. 1998, Thiele et al. 1998). Disruption of MC signaling leads to obesity and MC neurons appear to mediate the anorexic effects of the adipocyte hormone, leptin (Huszar et al. 1997, Seely et al. 1997). However, at least one potential difficulty in utilizing MC agonists as anorexic agents in humans is that the mixed MC agonist melanotan-II (MTII) reportedly produces a mild conditioned taste aversion (CTA) (Benoit et al. 2003). The CTA is thought to be caused by agents that produce adverse effects, like those of visceral illness. In other words, if rats are made ill after consumption of a novel flavor, then they avoid that flavor when encountered again, which is a phenomenon called a CTA. According to Benoit et al. (2003), the reduction in food intake caused by MTII is accompanied by CTA regardless of administration route.

In this study, we evaluated CKD-732's peripheral and central anti-obesity effects. To verify the mechanism of action of CKD-732 against obesity, we investigated the peripheral and central effects on body weight, food intake, and core temperature by subcutaneous (s.c.) or intracerebroventricular (i.c.v.) injection. The modulation of hypothalamic neuropeptide mRNAs after i.c.v. injection and the development of a CTA by s.c. injection were also evaluated to authenticate the mechanism for anorexia induced by CKD-732.

\section{Materials and methods}

\section{Animals}

Male obese animals such as arcuate nucleus (ARC) lesion mice, 7-week-old C57BL/6J ob/ob mice (Central Lab. Animal Inc., Seoul, South Korea), and 6-week-old Otsuka Long-Evans Tokushima fatty (OLETF) rats (Tokushima Research Institute Otsuka Pharmaceutical Co. Ltd, Tokushima, Japan) were obtained. Six-weekold C57BL/6J mice from the Jackson Laboratory (Daehan Biolink Co. Ltd, Seoul, South Korea), Sprague-Dawley (SD) rats (Daehan Biolink Co. Ltd), and Long-Evans Tokushima Otsuka (LETO) rats (Tokushima Research Institute Otsuka Pharmaceutical Co.) were also obtained and used as controls. These animals were maintained using a ratio of $12 \mathrm{~h}$ light: $12 \mathrm{~h}$ darkness cycle at $23{ }^{\circ} \mathrm{C}$ under the institutional guidelines for the humane treatment of laboratory animals. Mice and rats were housed with free access to food and water. PicoLab Rodent Diet 205053 (PMI Nutrition International, St Louis, MO, USA) was provided as their general rodent diet. After a 1-week acclimation, C57BL/6J mice and developed ARC lesion mice were administered s.c. injection for 7 days or single i.c.v. injection of CKD-732. The CKD-732 was also i.c.v. injected in C57BL/6J $o b / o b$ mice after 1-week acclimation. The SD rats and 18-week-old LETO and OLETF rats were administered s.c. injection of CKD-732 daily. All experimental protocols were approved by the Animal Ethics Committee at the Yonsei University College of Medicine (Seoul, South Korea).

\section{Drugs}

Monosodium glutamate (MSG), lithium chloride $(\mathrm{LiCl})$, and saccharin were purchased from Sigma. A synthetic TNP-470 analog, CKD-732 (6-O-(4-dimethylaminoethoxy) cinnamoyl fumagillol hemioxalate) was kindly provided by Chong Kun Dang Pharmaceutical, Seoul, South Korea. CKD-732 is known to be 1000 times more active than TNP-470 in vitro. Moreover, CKD-732 has been proven to be more effective in inhibition of human umbilical vein endothelial cells (HUVEC) growth and induction of cell cycle arrest in HUVEC when compared with TNP-470. The CKD-732 also significantly suppressed in vivo tumor growth, and its efficacy was much higher than TNP-470 (12). CKD-732 
was dissolved in $0.9 \% \mathrm{NaCl}$ and s.c. injected with doses of $0 \cdot 1,1,5$, or $10 \mathrm{mg} / \mathrm{kg}$ of body weight and i.c.v. injected with a single dose of $0.5,1,1.5,2,3$, or $5 \mu \mathrm{g} / 2 \mu \mathrm{l}$ artificial cerebrospinal final (aCSF) using the stereotaxic technique.

\section{Development of ARC lesion mice}

For the development of ARC lesions, pregnant C57BL/6J mice were housed alone before delivery. From days 1 to 7 , offspring were s.c. injected daily with either MSG ( $2 \mathrm{mg} / \mathrm{g}$ body weight) or $0.9 \% \mathrm{NaCl}$ (Spurlock et al. 1996). Animals were weaned at 21 days of age and then housed in groups of the same sex. All animals were allowed free access to water and standard rodent chow. Control and MSG-treated (ARC lesion) male mice were studied at 12 weeks of age. After death, their brains were removed and frozen with liquid nitrogen. The brains were later sectioned through the hypothalamus on a cryostat and the brain sections were fixed with paraformaldehyde. The sections were stained with Cresyl violet and then examined under a microscope to confirm the presence or absence of ARC lesions.

\section{Measurement of food intake, body weight, and core temperature}

Daily food intake was measured during 7 days of CKD-732 s.c. injection, and $4 \mathrm{~h}$ and $24 \mathrm{~h}$ after i.c.v. injection. Daily body weight was measured during 7 days of s.c. injection and until 2 weeks after CKD-732 withdrawal. Body weight change was also measured $24 \mathrm{~h}$ after i.c.v. injection. Core temperature was measured in fasted animals using a digital thermometer (Toshiba, Tokyo, Japan).

\section{Measurement of liver weights and regional fat contents}

LETO and OLETF rats were anesthetized with ketamine $(100 \mathrm{mg} / \mathrm{kg}$; Korea United Pharm., Seoul, South Korea) and xylazine $(10 \mathrm{mg} / \mathrm{kg}$; Bayer Korea Ltd). Rats were killed after aortic puncture and the liver and the epididymal, mesenteric, and perirenal fat pads were dissected, weighed, and frozen in liquid nitrogen.

\section{Measurement of the absolute number and size of adipocytes by histopathology}

The epididymal fat pads were fixed in $10 \%$ formalin neutral buffer $(\mathrm{pH} \mathrm{7.4)}$ and demineralized in nitric acid. Cut sections along with a perpendicular line of intercondylar fossa were embedded in paraffin. Sections $4 \mu \mathrm{m}$ thick were cut using a microtome, stained with hematoxylin and eosin, and examined under a light microscope with an image analyzer
(Image Pro, Silver Spring, MD, USA). Three cut sections of the same specimen were examined and the adipocytes were counted in each high power field (HPF) to calculate the absolute number and the size of adipocytes in each field.

\section{Reverse transcription-PCR (RT-PCR)}

To obtain the hypothalamus, the C57BL/6J and ARC lesion mice were killed $1 \mathrm{~h}$ after i.c.v. injection of CKD-732 at a dose of $3 \mu \mathrm{g} / 2 \mu \mathrm{l}$ aCSF. The hypothalami were dissected from decapitated animals and immediately frozen. Total RNA was extracted from the dissected hypothalamus with a commercial RNeasy kit (Qiagen) according to the manufacturer's instructions. The purity and the amount of isolated RNA were assessed by spectrophotometric measurement at 260 and $280 \mathrm{~nm}$. Total RNA $(5 \mu \mathrm{g})$ was reverse transcribed to cDNA at $42{ }^{\circ} \mathrm{C}$ for $50 \mathrm{~min}$ in a solution with a total volume of $20 \mu \mathrm{l}$ containing the following reagents: $0.5 \mathrm{mM}$ dNTP mixture, $10 \mathrm{mM}$ dithiothreitol, $0.5 \mathrm{mg}$ Oligo (dT), buffer (250 mM Tris, $375 \mathrm{mM} \mathrm{KCl}$, and $15 \mathrm{mM} \mathrm{MgCl}_{2}, \mathrm{pH} 8 \cdot 3$ ), and 5 U AMV (RNase H-free reverse transcriptase), all of which were obtained from Promega. The reaction was terminated at $70{ }^{\circ} \mathrm{C}$ for $15 \mathrm{~min}$. The cDNA aliquots were diluted in the ratio from 1:1 to 1:100. RT-PCR analysis was performed as previously described, under the following conditions $94{ }^{\circ} \mathrm{C}, 5 \mathrm{~min} ; 94^{\circ} \mathrm{C}, 30 \mathrm{~s} ; 52^{\circ} \mathrm{C}, 30 \mathrm{~s} ; 72{ }^{\circ} \mathrm{C}$, $1 \mathrm{~min}$ (repeated for 30 cycles); and $72^{\circ} \mathrm{C}, 15 \mathrm{~min}$. The following oligonucleotides were used for RT-PCR amplification: neuropeptide Y, (NPY); forward, 5'-CTAGGTAACAAGCGAATGGGG-3'; reverse, 5'-ACATGGAAGGGT CTTCAAGCC-3' ; agouti-related protein (AgRP), forward, 5'-TGCTGAGTTGTGTTCTGCTGT- ${ }^{\prime}$; reverse, $5^{\prime}$-AGT GGACTCAGGTCAGGGA-3'; pro-opiomelanocortin, (POMC); forward, 5' -AACAGCAGCAGTGCTGGCA-3'; reverse, 5'-AAGTGCTCCACCCGGTAGG-3'; melanin concentrating hormone, $(\mathrm{MCH})$; forward, $5^{\prime}$-GCAAAG ATGACTCTCTCTTCC-3'; reverse, 5'-GACTTGCCAACATGGTCGGTA-3'; $\beta$-actin, forward, $5^{\prime}$-TTCAACACCCCAGCCATGT-3'; reverse, 5'-TGTGGTACG ACCAGAGGCATAC-3'

\section{Analysis of a CTA}

$\mathrm{LiCl}$ is commonly used as a toxic agent to produce a CTA. In order to test CTA, a modified method from Benoit $e t a l$. (2003) was used. OLETF and LETO rats were first habituated to $1 \mathrm{~h}$ access to water. For 7 days, tap water was replaced with water containing $0 \cdot 1 \%$ saccharin. Then, the rats were given $1 \mathrm{~h}$ access to tap water for 2 days. These rats received a single s.c. injection of either saline, $\mathrm{LiCl}$ $(0 \cdot 15 \mathrm{M}$ at $2 \%$ body weight), or two different doses of CKD-732 ( 1 or $10 \mathrm{mg} / \mathrm{kg}$ in a volume of $2 \mathrm{ml} / \mathrm{kg}$ ) just after $1 \mathrm{~h}$ exposure to the saccharin solution. On the 
following day, the rats again received $1 \mathrm{~h}$ access to two bottles of tap water. On the subsequent day, a two-bottle choice test was administered in which all rats were given $1 \mathrm{~h}$ access to tap water and the saccharin solution, with the relative position of the two solutions counterbalanced across subjects. The preference ratio (saccharin solution intake divided by total fluid intake) was calculated from the results of the final experimental day.

\section{Statistical analysis}

All parameters were analyzed by SAS V8.1 (Cary, NC, USA). All values were reported as means \pm s.D. Statistical significance was determined by using the Student's $t$-test and one-way ANOVA followed by Tukey's post hoc test. A value of $P<0.05$ was taken to indicate statistical significance.

\section{Results}

\section{Effect of CKD-732 on food intake, body weight, and core temperature}

To investigate the in vivo effect of CKD-732 on food intake and body weight, CKD-732 was s.c. injected for
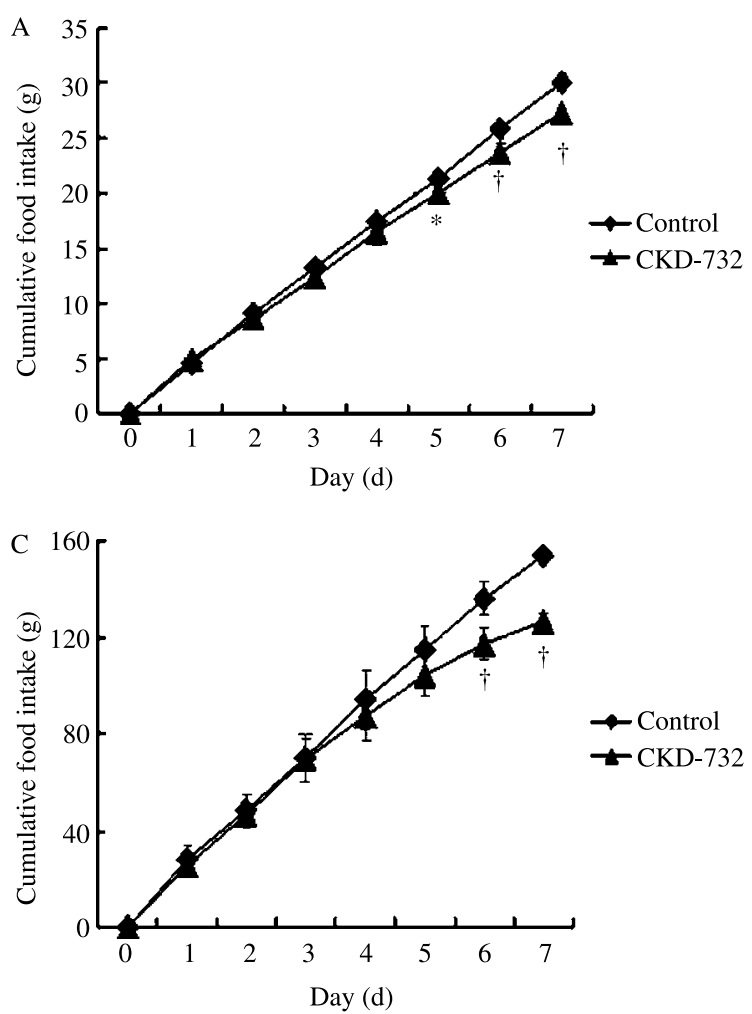

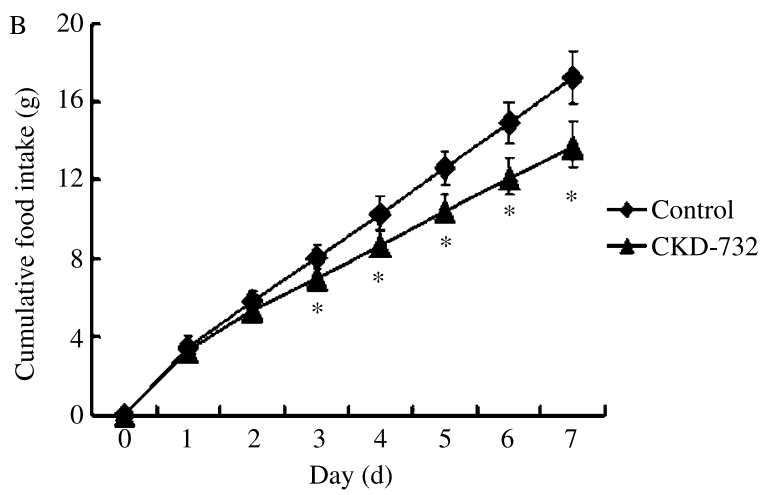

7 days at different doses: C57BL/6J mice, $5 \mathrm{mg} / \mathrm{kg}$ per day; ARC lesion mice, $1 \mathrm{mg} / \mathrm{kg}$ per day; SD rats, $5 \mathrm{mg} / \mathrm{kg}$ per day; LETO rats, $1 \mathrm{mg} / \mathrm{kg}$ per day; and OLETF rats, $1 \mathrm{mg} / \mathrm{kg}$ per day. Seven days of CKD-732 s.c. injection decreased cumulative food intake in all animals, but to a greater extent in obese models including the ARC lesion mice and OLETF rats (e.g. $114 \cdot 8 \pm 13 \cdot 4 \mathrm{~g}$ for treated OLETF rat versus $170 \cdot 7 \pm$ $20.6 \mathrm{~g}$ for control OLETF rat, $P<0.01$ by Student's $t$ test, Fig. 1). Accordingly, these animals demonstrated a significant decrement in body weight; up to a $44 \pm 3 \mathrm{~g}$ decrease was shown in OLETF rats with only 7 days of CKD-732 injection, and whose basal mean body weight was $567 \cdot 8 \pm 16.6 \mathrm{~g}$ (Fig. 2). In other words, a $7 \cdot 9 \pm 0 \cdot 5 \%$ body weight decrease was obtained in treated OLETF rats when compared with a $0 \cdot 3 \pm 2 \cdot 2 \%$ decrease in control OLETF rats within 7 days of treatment $(P<0 \cdot 01$ by Student's $t$-test).

To investigate CKD-732's effect on energy expenditure, we measured the core temperature of fasted ARC lesion mice after 7 days of CKD-732 treatment. The core temperature of CKD-732-treated ARC lesion mice was greater than saline-treated ARC lesion mice, which suggests an increase of energy expenditure upon CKD732 treatment (Fig. 3A).

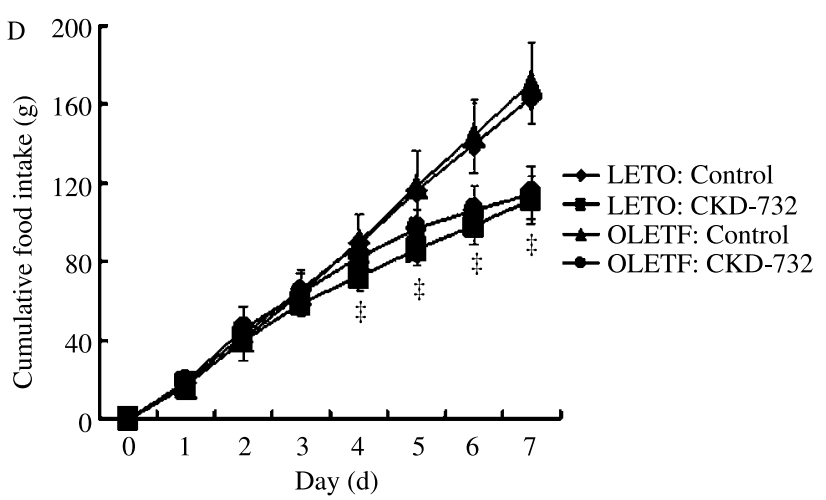

Figure 1 Cumulative food intake by CKD-732 s.c. injection; $n=20$ per each animal model. (A) C57BL/6J mouse, (B) ARC lesion mouse, (C) SD rat, and (D) LETO/OLETF rat. ${ }^{*} P<0.05$; ${ }^{\dagger} P<0.01$ vs control by Student's $t$-test. ${ }^{\ddagger} P<0.05$ by one-way ANOVA followed by Tukey's post hoc analysis. 

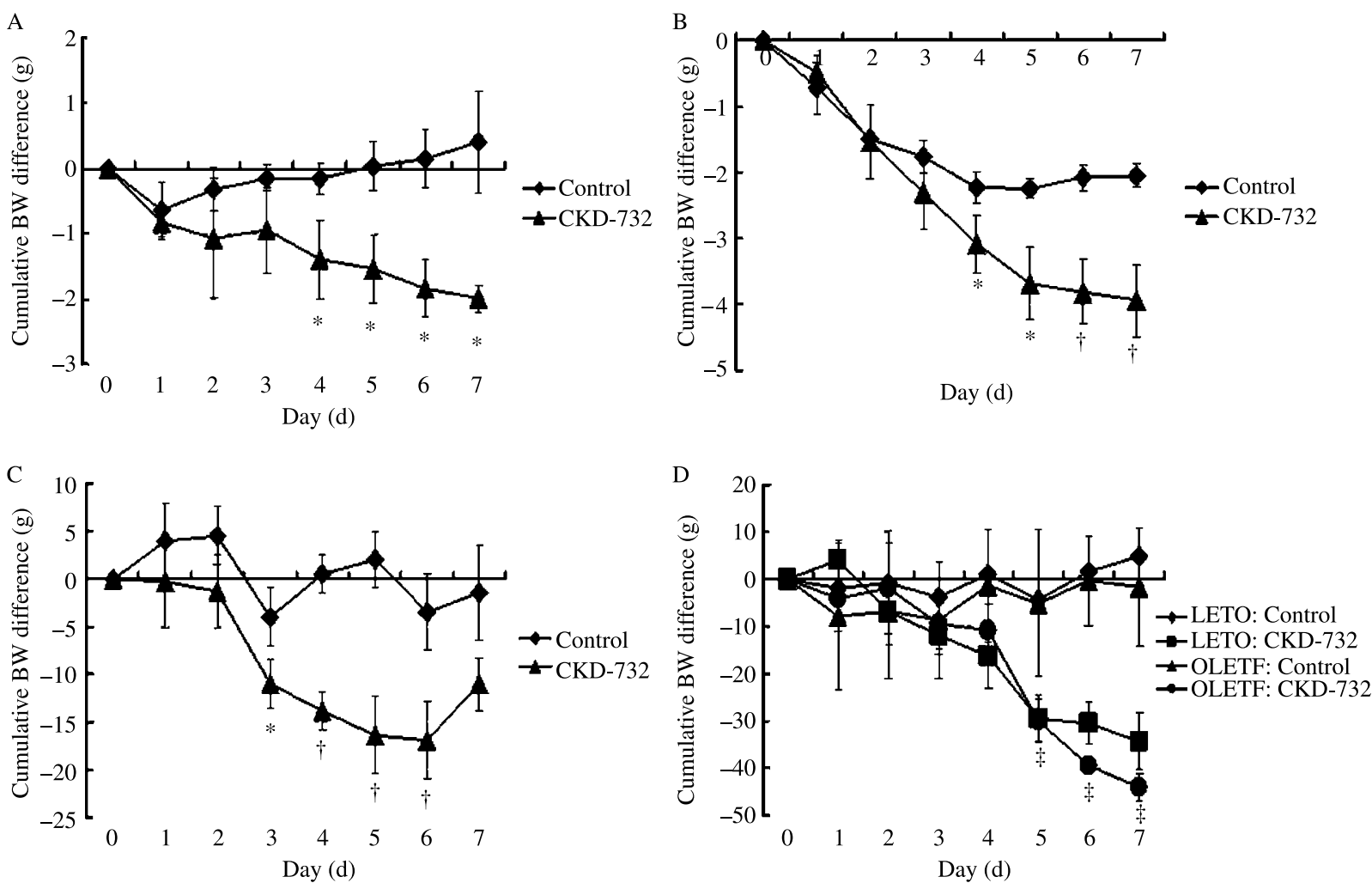

Figure 2 Body weight (BW) change by CKD-732 s.c. injection; $n=20$ each per animal model. (A) C57BL/6J mouse, (B) ARC lesion mouse, (C) SD rat, and (D) LETO/OLETF rat. ${ }^{\star} P<0.05 ;{ }^{\dagger} P<0.01$ vs control by Student's $t$-test. ${ }^{\ddagger} P<0.05$ by one-way ANOVA followed by Tukey's post hoc analysis.

In addition, we examined the reversibility of body weight after CKD-732 withdrawal in ARC lesion mice. We found that if CKD-732 treatment was discontinued after 7 days of s.c. injection, body weight slowly returned to the basal level within 2 weeks (Fig. 3B). This result suggests the idea of using repetitive on and off treatment to minimize weight cycling and maximize long-term weight maintenance.

When CKD-732 was s.c. injected at a higher dosage $(10 \mathrm{mg} / \mathrm{kg})$ for 7 days, LETO and OLETF rats experienced anorexia from the first day of treatment and their body weight significantly decreased from the second day of treatment (Fig. 3C and D). This rapid induction of anorexia was similarly observed in both LETO and OLETF rats.

\section{Effect of CKD-732 on liver weights, regional fat contents, and size of adipocytes}

To investigate CKD-732's effect on liver weights, regional fat contents, and size of adipocytes, LETO and OLETF rats were killed after 7 days of CKD-732 treatment. Weights of the liver were not significantly different between CKD732 -treated and control LETO rats $(14 \cdot 0 \pm 0 \cdot 3 \mathrm{~g} /$ rat vs
$14 \cdot 4 \pm 0 \cdot 2 \mathrm{~g} / \mathrm{rat}, P=0 \cdot 134)$, and CKD-732-treated and control OLETF rats $(19 \cdot 2 \pm 0 \cdot 3 \mathrm{~g} / \mathrm{rat}$ vs $19 \cdot 7 \pm 0 \cdot 3 \mathrm{~g} / \mathrm{rat}$, $P=0 \cdot 065)$. In CKD-732-treated LETO and OLETF rats, regional fat pad weights, especially epididymal and mesenteric fat pads, were significantly decreased (Fig. 4). Moreover, by histopathologic examination, the number $(n)$ and size $\left(\mu^{2}\right)$ of adipocytes per HPF were calculated. As shown in Fig. 5, the size of adipocytes decreased significantly in CKD-732-treated LETO and OLETF rats. However, the change in endothelial cells could not be evaluated in these cut sections due to stain method differences. In addition, we found no evidence of adipose tissue necrosis by microscopic evaluation. From these results, although the precise mechanism is still unknown, we suggest that the antiangiogenic signal induced by CKD-732 directly influences the adipose tissue to shrink significantly, and as a consequence, adipocyte size decreases.

\section{Mechanism of action of CKD-732 on reducing body weight and food intake}

To determine how CKD-732 reduces body weight and food intake, we evaluated the central anorexic effect of 


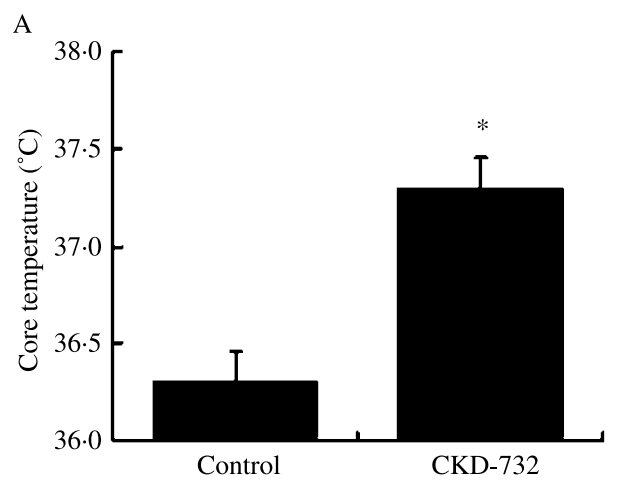

ARC lesion mice

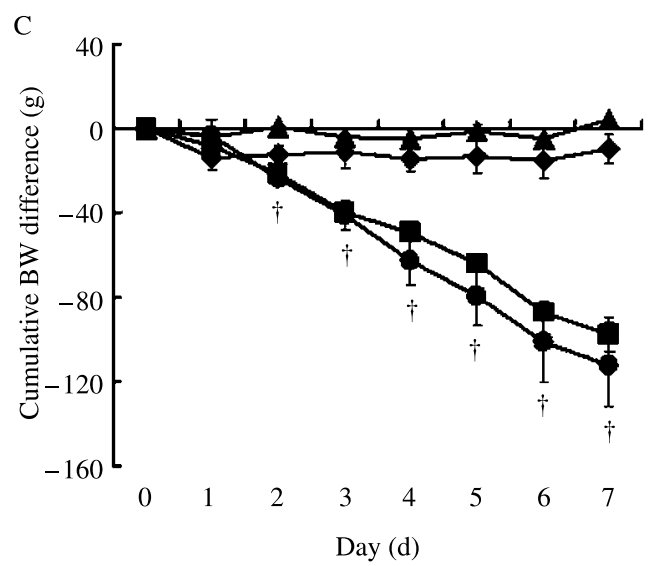

B

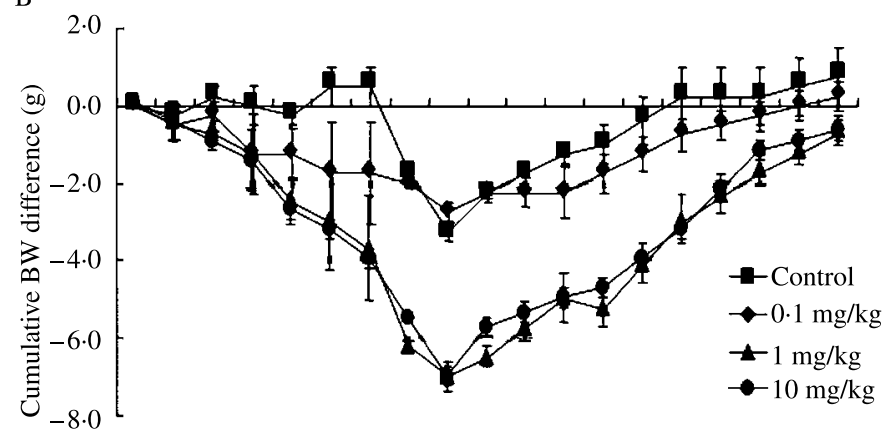

T0 T1 T2 T3 T4 T5 T6 P0 P1 P2 P3 P4 P5 P6 P7 P8 P9 P10 P11 Day (d)

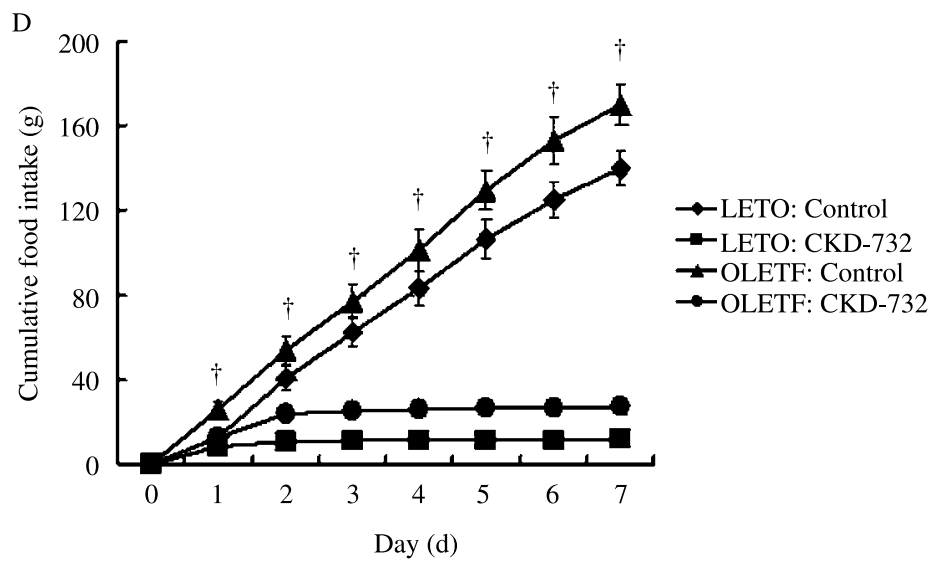

Figure 3 Effect on core temperature and change of body weight (BW) after CKD-732 treatment withdrawal, and high-dosage effects. (A) The core temperature of ARC lesion mice was significantly increased after 7 days of CKD-732 s.c. injection; $n=10$ per each subgroup. ${ }^{\star} P<0.05$ vs control by Student's $t$-test. (B) Reversible body weight recovery after withdrawal of CKD-732 treatment in ARC lesion mice ( $n=5$ per each subgroup). T0-T6 indicates treatment days and P0-P11 indicates post-treatment days. (C) and (D) Cumulative body weight difference and food intake of LETO $(n=20)$ and OLETF rats $(n=20)$ when $10 \mathrm{mg} / \mathrm{kg}$ dose of CKD-732 was s.c. injected for 7 days. ${ }^{\dagger} P<0.001$ by one-way ANOVA.

CKD-732 in animal models. We injected CKD-732 i.c.v. at a single dose of $0 \cdot 5,1,1 \cdot 5,3$, or $5 \mu \mathrm{g} / 2 \mu \mathrm{l}$ aCSF using the stereotaxic technique in ARC lesion mice and normal littermates. The validity of a single i.c.v. injection by the stereotaxic technique was proven by examining the stained ventricle areas with Toluidine blue, shortly after i.c.v. injection. Body weight, food intake, and core temperature was measured 4 and $24 \mathrm{~h}$ after CKD-732 i.c.v. injection. Although anorexic effects were not found in normal littermates, the $24 \mathrm{~h}$ body weight and 4 and $24 \mathrm{~h}$ food intakes were significantly decreased in a dose-dependent manner in ARC lesion mice (Table 1). However, the core temperature was not altered in ARC lesion mice and normal littermates by a single central injection of CKD-732 (Table 1).

To establish the central action of CKD-732 in different obesity models, $2 \mu \mathrm{g} / 2 \mu \mathrm{l}$ CKD-732 were i.c.v. injected in five $o b / o b$ mice and $2 \mu \mathrm{laCSF}$ was i.c.v. injected in another five $o b / o b$ mice as controls. The basal body weights of $o b / o b$ mice were not significantly different: $36 \cdot 2 \pm 0.72 \mathrm{~g}$ vs
$36 \cdot 4 \pm 0 \cdot 55 \mathrm{~g}$ (CKD-732 group versus control group; $P=0.88$ by Student's $t$-test). Like the results in Table 1,4 and $24 \mathrm{~h}$ food intakes were significantly lower in the CKD732 group: $0 \cdot 62 \pm 0 \cdot 19 \mathrm{~g}$ vs $1 \cdot 35 \pm 0 \cdot 15 \mathrm{~g}$ and $4 \cdot 30 \pm 0 \cdot 39 \mathrm{~g}$ vs $5 \cdot 84 \pm 0 \cdot 37 \mathrm{~g}$ (CKD-732 group versus control group; $P<0.05$ by Student's $t$-test respectively). The change in body weight during $24 \mathrm{~h}$ after single i.c.v. injection was significantly different: $-0 \cdot 38 \pm 0 \cdot 34 \mathrm{~g}$ vs $0 \cdot 96 \pm 0 \cdot 19 \mathrm{~g}$ (CKD-732 group versus control group; $P<0.05$ by Student's $t$-test).

To verify the changes in hypothalamic neuropeptide mRNAs when CKD-732 was centrally injected, we killed ARC lesion mice and normal littermates $1 \mathrm{~h}$ after i.c.v. injection. The basal POMC and NPY mRNA expression levels in ARC lesion mice were decreased to 41 and $39 \%$ of normal littermates respectively. However, all POMC, NPY, AgRP, and MCH mRNA expressions were not significantly altered by CKD-732 i.c.v. injection in ARC lesion mice and normal littermates beyond our expectation (Fig. 6). 

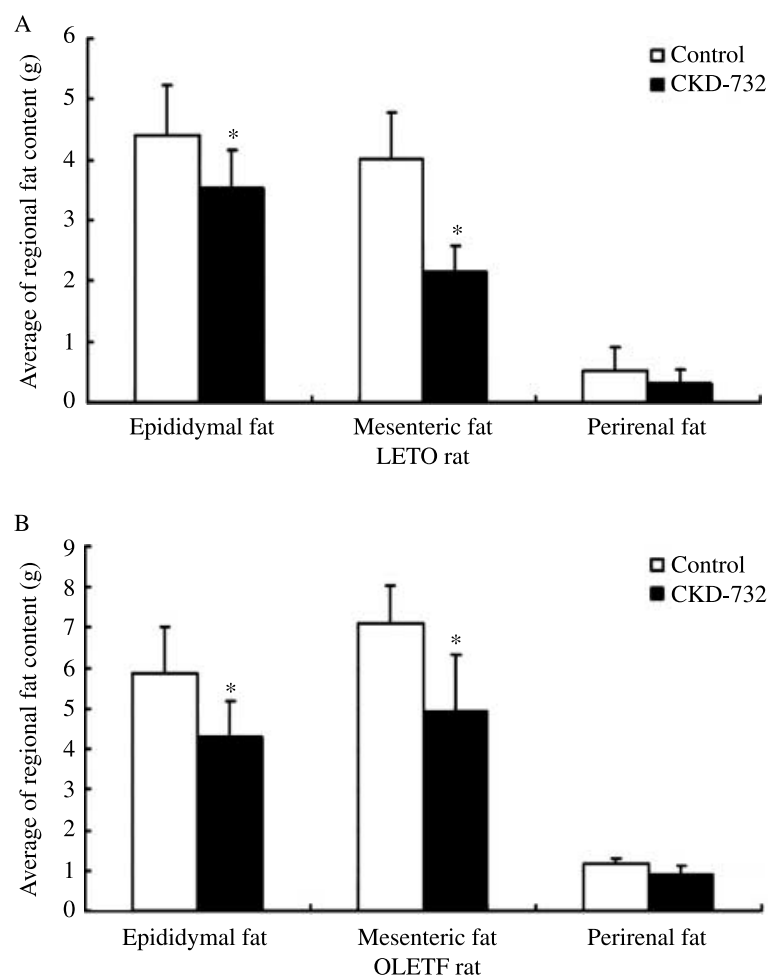

Figure 4 Reduction of regional fat contents after 7 days of CKD-732 s.c. injection. Regional fat contents of (A) LETO $(n=10$ per subgroup) and (B) OLETF ( $n=10$ per subgroup) rats are shown. ${ }^{\star} P<0.05$ vs control by Student's $t$-test.

To further study the action mechanism of CKD-732 on food intake control, we checked for CTA by a single peripheral injection of CKD-732. LETO and OLETF rats with saline injection exhibited no preference for saccharin flavor. In contrast, the rats in both the $\mathrm{LiCl}$ and CKD-732-treated groups significantly preferred the neutral flavor, indicating the development of CTA. In OLETF rats, the preference ratio was $85.4 \%$ for control, $7.9 \%$ for $\mathrm{LiCl}$ treated, $12.1 \%$ for CKD-732 $1 \mathrm{mg} / \mathrm{kg}$ treated, and $7 \cdot 2 \%$ for $10 \mathrm{mg} / \mathrm{kg}$ treated rats $(P<0.01 \mathrm{for}$ $\mathrm{LiCl}, 1 \mathrm{mg} / \mathrm{kg}$ treated, and $10 \mathrm{mg} / \mathrm{kg}$ treated vs control). Additionally, a CTA produced by administration of CKD732 was found to be dose-dependent and more easily achieved in OLETF than LETO rats (Fig. 7). These results suggest that CKD-732 acutely causes CTA, which may contribute to decreased food intake caused by CKD-732, especially in obese models.

\section{Discussion}

To overcome the limitations of obesity therapy, an angiogenesis inhibitor has recently been speculated as a potential anti-obesity drug (Rupnick et al. 2002). Likewise, Brakenhielm et al. (2004a) have recently proved the preventive effect of the angiogenesis inhibitor, TNP-470, diet-induced obese models. They showed that TNP-470 decreased serum levels of low-density lipoprotein cholesterol and insulin levels, and increased insulin sensitivity (Brakenhielm et al. 2004a). According to their results, TNP-470 could be useful in preventing obesity and ameliorating insulin resistance, which is a major causative factor for type 2 diabetes mellitus (Elmquist \& Marcus 2003). However, the mechanism of such novel drugs on body weight and food intake control, or metabolic adaptation, is largely unknown. In order to verify the anti-obesity action mechanism of the TNP-470 analog, CKD-732, we evaluated: 1) changes in body weight, food intake, and core temperature $1 \mathrm{~h}$ after a single i.c.v. injection, 2) alteration of hypothalamic anorexic and orexigenic neuropeptide expression $1 \mathrm{~h}$ after a single i.c.v. injection, and 3) induction of a CTA by a single s.c. injection.

In this study, we demonstrated that CKD-732 significantly reduced the body weight and food intake in obese and non-obese animal models by peripheral injection. Previous studies have shown that various angiogenesis inhibitors have peripheral anti-obesity effects and prevent obesity with decreased insulin resistance mainly in obese animals, as non-obese models were not affected by these agents (Rupnick et al. 2002, Brakenhielm et al. 2004a). Apart from previous reports, our findings included different types of obesity models such as ARC lesion mouse, whose ARC nucleus was ablated by neonatal MSG injection, and OLETF rat, which is a model for type 2 diabetes mellitus and obesity. Moreover, we suggest a modest but similar pattern of CKD-732's anti-obesity effects in non-obese models. In terms of animal models, ARC lesion mice are known to represent hypophagic, hypometabolic, obese mice, and $o b / o b$ mice are known as leptin-deficient, hyperphagic obese mice, whereas OLETF rats become obese and develop diabetes mellitus due to hyperphagia. We experienced similar anti-obesity effects of CKD-732 in these specific obese models, in that CKD-732 is effective in both hypometabolic and hyperphagic obesity. These effects were induced within relatively short periods such as 7 days after s.c. CKD-732 injection. After death, the regional fat contents of epididymal and mesenteric fat pads were significantly decreased in CKD-732-treated OLETF and LETO rats. When we evaluated the histopathology of these shrunken fat pads, we found that the absolute adipocyte number in each HPF was higher, and thus adipocyte size was significantly decreased in CKD-732-treated rats. Although we could not define endothelial cells in the fat pads, the decrease of the size of adipocytes could be surmised by the selective apoptosis of endothelial cells in adipose tissue and shrinkage of existing adipocytes, as presented previously (Lucas et al. 1998, Dhanabal et al. 1999, Rupnick et al. 2002, Brakenhielm et al. 2004b). Through these results, we strongly suggest that CKD-732's direct 
$5-1$
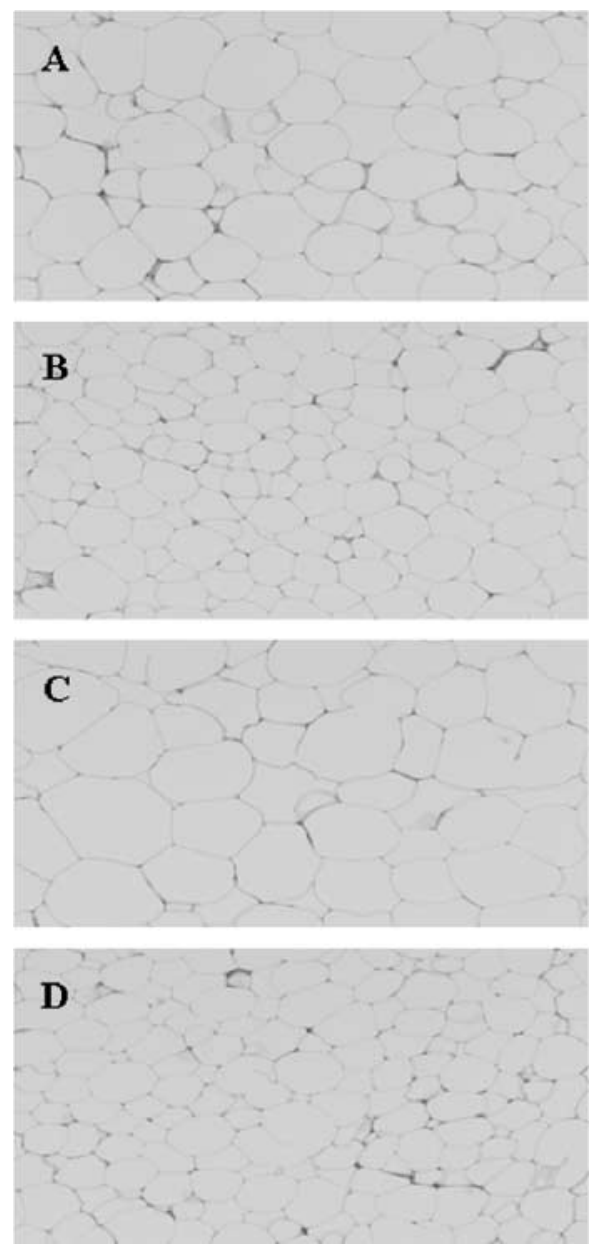

$5-2$
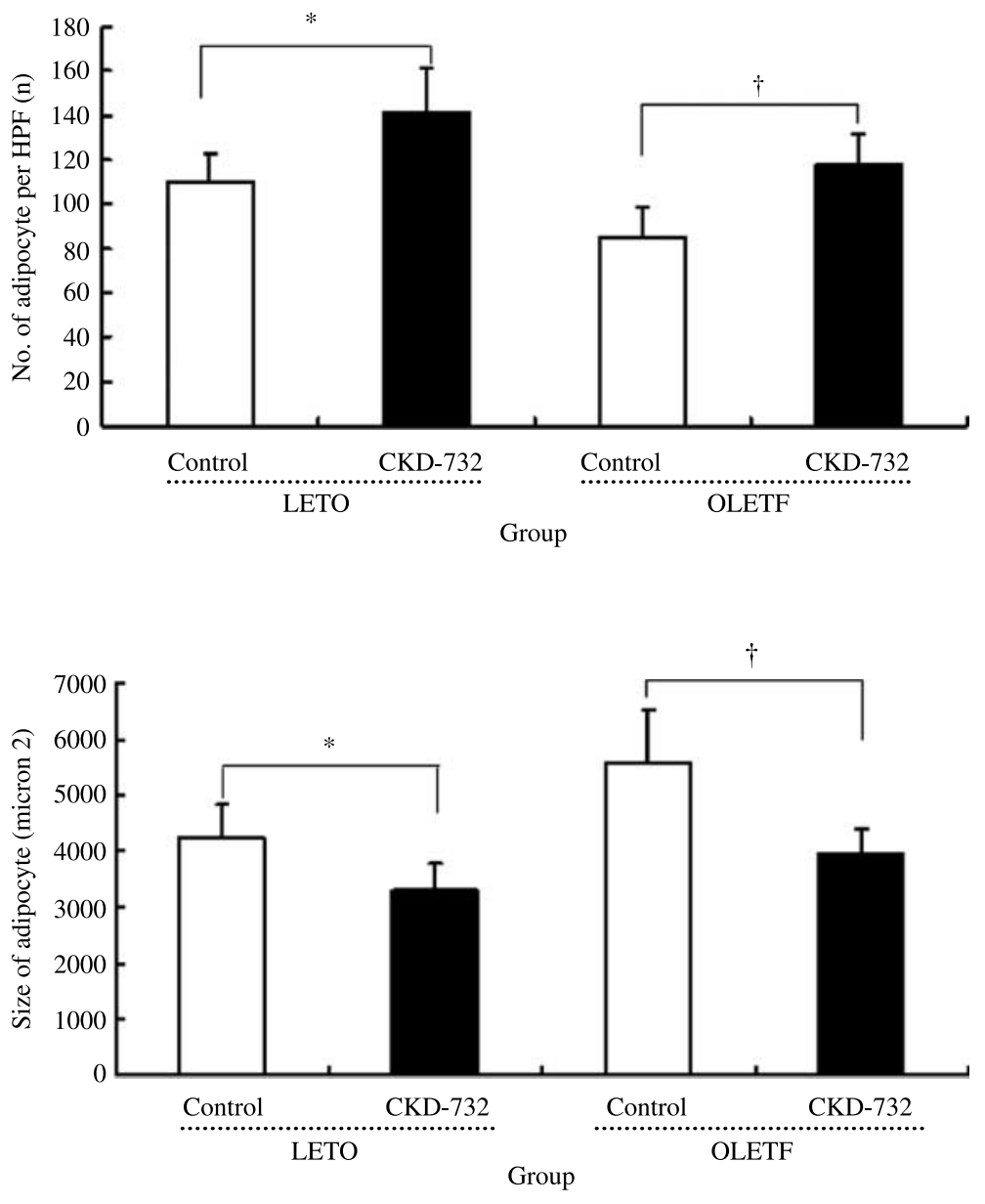

Figure 5 Histopathology of epididymal fat in OLETF and LETO rats after 7 days of CKD-732 s.c. injection. (5-1) H-E stain of epididymal fat is shown $(\times 200)$. (A) LETO control, (B) LETO CKD-732 treated, (C) OLETF control, (D) OLETF CKD-732 treated. (5-2) CKD-732 increased the number $(n)$ of adipocytes per HPF and decreased the adipocyte size $\left(\mu^{2}\right)$ significantly. ${ }^{\star} P<0 \cdot 05 ;{ }^{\dagger} P<0 \cdot 01$ vs control by Student's $t$-test.

stimulation of adipose tissue contributes to one part of an anti-obesity mechanism causing significant shrinkage of adipose tissue and subsequent body weight reduction.

Although TNP-470 is a selective angiogenesis inhibitor, in clinical trials for the treatment of cancer, high dosages of this agent may affect other systems in the body. Thus, we cannot exclude the possibility that CKD-732 may modify obesity through mechanisms other than antiangiogenesis. For example, CKD-732 affects food intake, even though this reduction seems to

Table 1 Change of body weight, food intake, and core temperature 4 and $24 \mathrm{~h}$ after CKD-732 intracerebroventricular (i.c.v.) injection

\begin{tabular}{|c|c|c|c|c|c|c|c|c|}
\hline & \multicolumn{4}{|c|}{ C57BL/6J mouse ( $n=5$ per each subgroup) } & \multicolumn{4}{|c|}{ ARC lesion mouse ( $n=5$ per each subgroup) } \\
\hline & aCSF & $1 \cdot 0 \mu \mathrm{g}$ & $3.0 \mu \mathrm{g}$ & $5 \cdot 0 \mu \mathrm{g}$ & aCSF & $0.5 \mu \mathrm{g}$ & $1.0 \mu \mathrm{g}$ & $1.5 \mu \mathrm{g}$ \\
\hline $24 \mathrm{~h} \mathrm{BW}$ change $(\mathrm{g})$ & $0.36 \pm 0.07$ & $0.26 \pm 0.22$ & $0.22 \pm 0.23$ & $0.25 \pm 0.07$ & $-0.57 \pm 0.08$ & $-0.72 \pm 0.05$ & $-0.87 \pm 0.16$ & $-1 \cdot 05 \pm 0.15^{\star}$ \\
\hline $4 \mathrm{~h}$ food intake $(\mathrm{g})$ & $1.03 \pm 0.02$ & $1.22 \pm 0.03$ & $0.99 \pm 0.18$ & $1 \cdot 11 \pm 0 \cdot 14$ & $0.64 \pm 0.01$ & $0.57 \pm 0.03$ & $0.43 \pm 0.02^{\dagger}$ & $0.25 \pm 0.0$ \\
\hline $24 \mathrm{~h}$ food intake $(\mathrm{g})$ & $3.97 \pm 0.11$ & $4 \cdot 13 \pm 0.01$ & $4 \cdot 17 \pm 0.33$ & $4.02 \pm 0.33$ & $2 \cdot 09 \pm 0.18$ & $2 \cdot 34 \pm 0.11$ & $1 \cdot 84 \pm 0 \cdot 16$ & $1 \cdot 30 \pm 0.25^{\star}$ \\
\hline Core temperature $\left({ }^{\circ} \mathrm{C}\right)$ & $37 \cdot 37 \pm 0.11$ & $37 \cdot 50 \pm 0.18$ & $37 \cdot 38 \pm 0 \cdot 10$ & $37.54 \pm 0.09$ & $37 \cdot 44 \pm 0.09$ & $37.55 \pm 0.08$ & $37 \cdot 84 \pm 0.04$ & $37 \cdot 82 \pm 0.04$ \\
\hline
\end{tabular}

${ }^{\star} P<0.05 ;{ }^{\dagger} P<0.01$ vs control (aCSF i.c.v. injected mice of the same group) by one-way ANOVA. 
$\begin{array}{llll}\text { A } & \text { B } & \text { C } & \text { D }\end{array}$

A
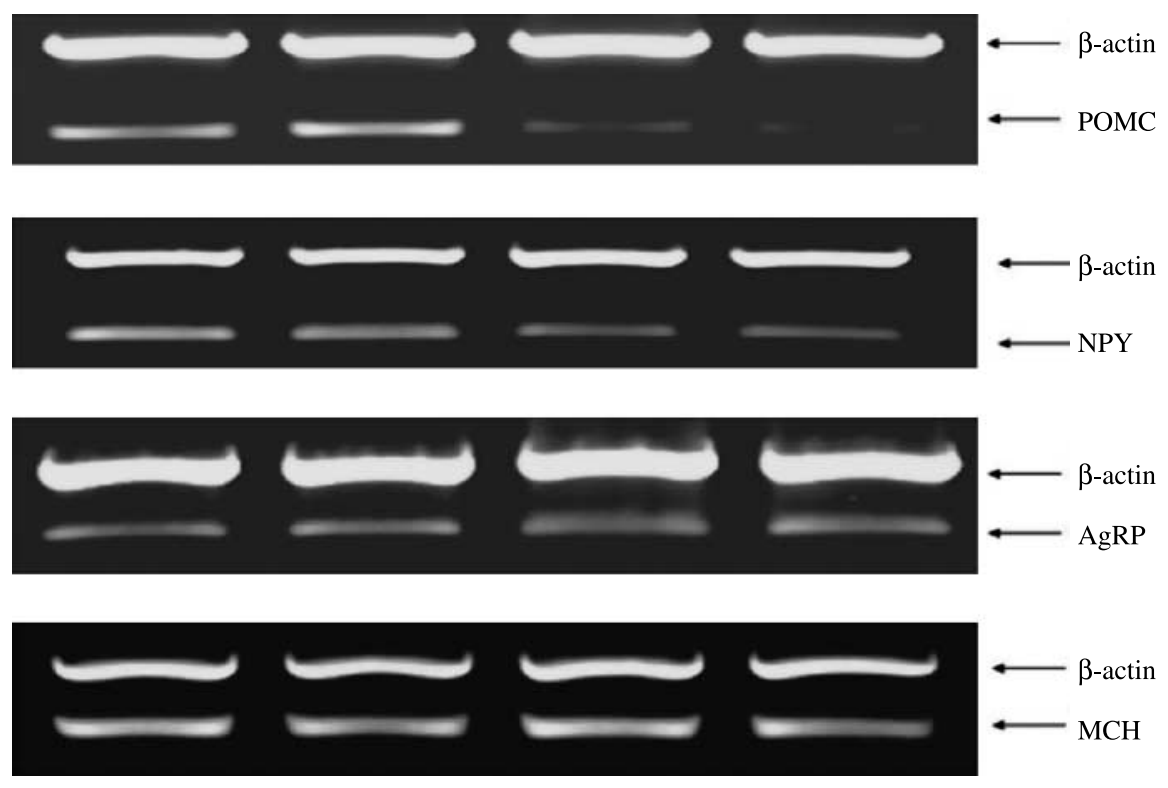

B
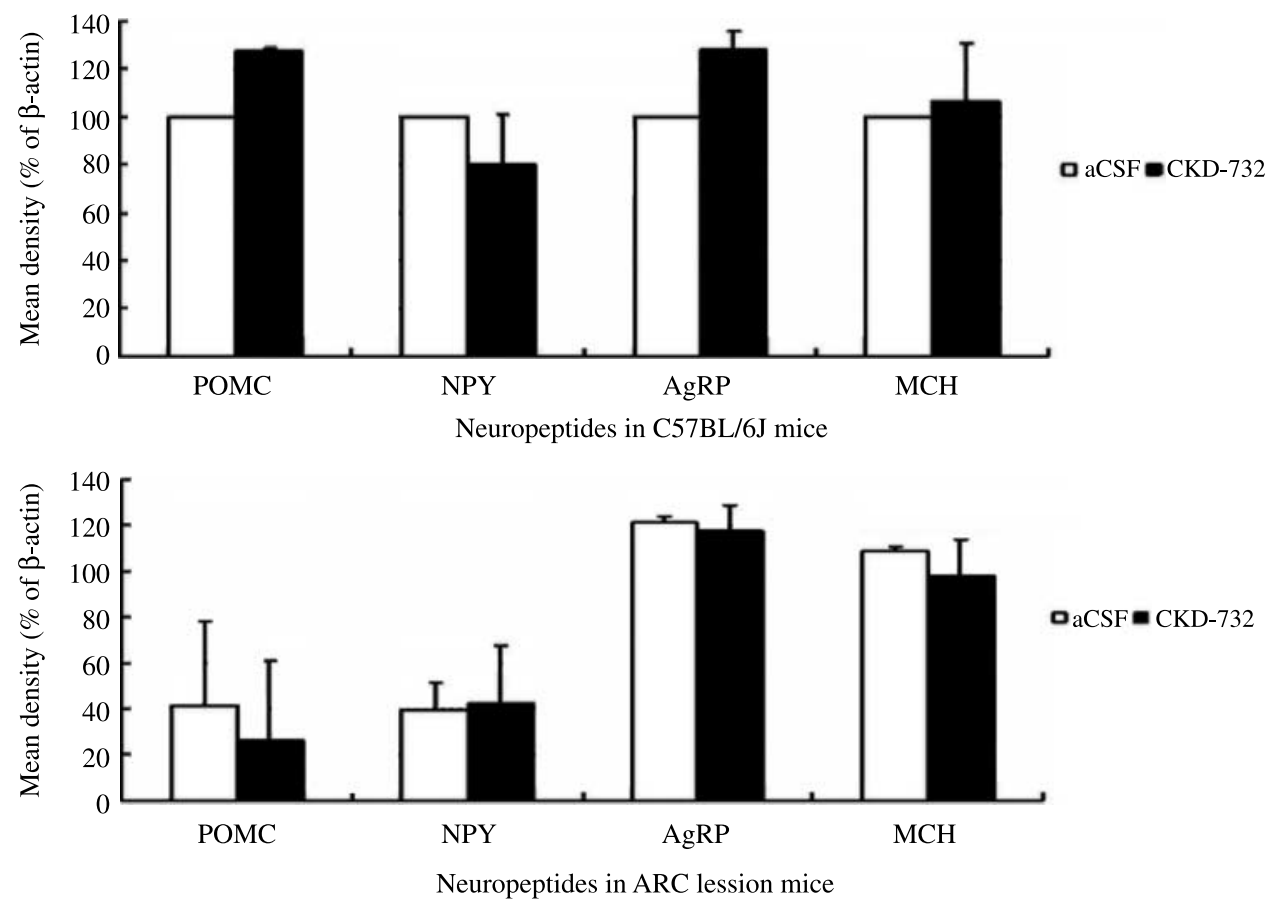

Figure 6 RT-PCR of hypothalamic neuropeptide mRNA $1 \mathrm{~h}$ after single i.c.v. injection of CKD-732. C57BL/6J mice ( $n=5$ per subgroup), (A) control and (B) CKD-732 treated. ARC lesion mice ( $n=5$ per subgroup), (C) control and (D) CKD-732 treated. No significant neuropeptide mRNA mobilization was observed.

mostly affect obese models, with a modest effect in nonobese models. In this regard, although the body weight and food intake of normal littermates were not altered despite increment of the drug concentration, the body weight and food intake of ARC lesion mice and $o b / o b$ mice were decreased with a relatively lower dosage than that of normal littermates. Several reasons may explain why this central effect was only presented in obese mice, 

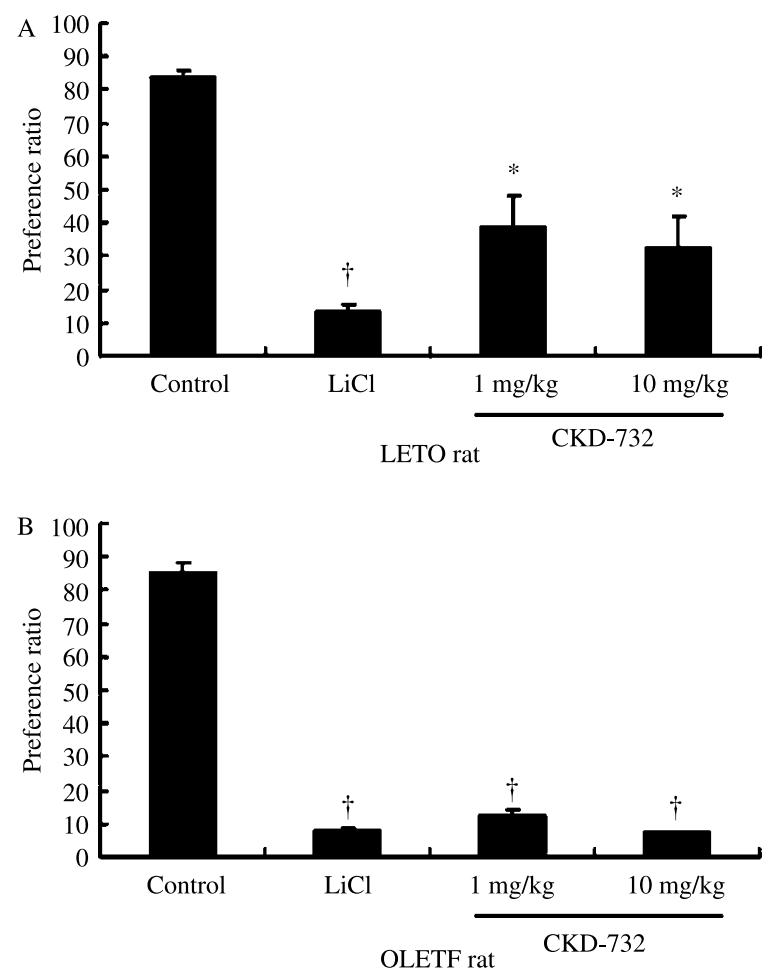

Figure 7 Conditioned taste aversion induced by CKD-732 single s.c. injection. (A) LETO ( $n=10$ per subgroup) and (B) OLETF rats ( $n=10$ per subgroup). CKD-732-treated OLETF and LETO rats favored neutral flavor, which was more profoundly demonstrated in OLETF rats. ${ }^{\star} P<0.05 ;{ }^{\dagger} P<0.01$ vs control by one-way ANOVA followed by Tukey's post hoc analysis.

one of which might be the higher permeability of the blood-brain barrier in obese models (including ARC lesion mice and $o b / o b$ mice) when compared with normal models (Skultetyova et al. 1998). Several experiments have suggested that blood-brain barriers of obese models, such as ARC lesion mice and $o b / o b$ mice, are more permeable than normal animals, especially under stress conditions (Sharma \& Dey 1988, Skultetyova et al. 1998, Peruzzo et al. 2000). A second explanation for this consequence might be the different effective dosages needed for different species or models, as food intake is regulated according to body size and drug sensitivity. Accordingly, we propose that an indirect central action of CKD-732 partly account for reduction of food intake in such animal models.

In terms of regulating energy homeostasis, food intake and energy balance is influenced by well-known hypothalamic orexigenic or anorexigenic neuropeptides (Woods et al. 1998). We demonstrated that a single i.c.v. injection of CKD-732 induced no significant increase or decrease in various neuropeptides in ARC lesion mice or in normal littermates. As previously shown, the basal mRNA expression of NPY and POMC in ARC lesion mice was decreased to about $40 \%$ of that of normal littermates, which indicates that the selective ablation of the ARC nucleus may result in a significant decrease of both orexigenic and anorexigenic signals (Broberger et al. 1998). However, taken together, these data indicate that the action point of CKD-732 in the hypothalamus does not affect the transcriptional level of neuropeptides. Additional experiments that evaluate CKD-732's unknown supplementary central mechanism, such as its regulation of translational or post-translational modification of hypothalamic neuropeptides, and that of secretion or stability of various neuropeptides need to be performed in the near future.

In developing novel drugs for obesity, some agents induce unexpected phenomena, which may limit the use of such agents. Recently, MC studies have shown that CTA, which is the induction of an abnormal taste or visceral illness such as nausea or gastrointestinal discomfort, consequently may decrease food intake (Abe et al. 1994, Benoit et al. 2003). Moreover, it has been shown that the reduction of food intake caused by MTII was accompanied by adverse consequences such as a mild CTA, regardless of administration route. Likewise, we experienced a similar CTA by peripheral injection of CKD-732 in OLETF and LETO rats that was as significant as $\mathrm{LiCl}$ injection. These effects were shown to be dose-dependent and more profoundly affected OLETF rats. This result suggests that anorexia caused by peripheral injection can be partly explained by the induction of a CTA. However, avoidance of a specific taste induced by CTA does not represent a direct decrease in appetite. Besides, the influence of CKD-732 on food intake can only partially explain its anti-obesity effect because pair-fed control animals were significantly more obese than the TNP-470-treated obese models, as previously shown (Rupnick et al. 2002). Therefore, we suggest that the TNP-470 analog, CKD732 , has a multifunctional action that induces direct shrinkage of adipose tissue and anorexia caused by direct hypothalamic stimuli or a CTA.

This study has some limitations that should be addressed in further studies. First, CKD-732 was the only type of angiogenesis inhibitor we used to evaluate the anti-obesity effect in our study. Secondly, we included specific models of obesity, such as ARC lesion mice, $o b / o b$ mice, and OLETF rats, which may limit the wide application of such results. Finally, we only used mouse models when performing i.c.v. injection and performed a single central stereotaxic injection instead of a continuous central infusion using cannula with an osmotic pump when searching for precise regulation of hypothalamic neuropeptides.

In conclusion, our findings provide some insight into the mechanism of action of the angiogenesis inhibitor, 
CKD-732 on obesity control. CKD-732 was found to decrease food intake, body weight, fat mass, and the size of adipocytes. Moreover, although it was only proven in selected animal models, CKD-732 induced central anorexia and a CTA. The results of this study should be carefully referred to and verified when developing CKD-732 for anti-obesity or anti-cancer treatments.

\section{Acknowledgements}

This work was supported by research grants from the Korea Ministry of Health and Welfare (03-PJ1-PG1CH05-0005) and by the Brain Korea 21 Project for Medical Sciences, Yonsei University. The authors declare that there is no conflict of interest that would prejudice the impartiality of this scientific work.

\section{References}

Abe J, Zhou W, Takuwa N, Taguchi J, Kurokawa K, Kumada M \& Takuwa Y 1994 A fumagillin derivative angiogenesis inhibitor, AGM1470, inhibits activation of cyclin-dependent kinases and phosphorylation of retinoblastoma gene product but not protein tyrosyl phosphorylation or protooncogene expression in vascular endothelial cells. Cancer Research 54 3407-3412.

Benoit SC, Sheldon RJ, Air EL, Messerschmidt P, Wilmer KA, Hodge KMB, Jones MB, Eckstein DMM, McOsker CC, Woods SC et al. 2003 Assessment of the aversive consequences of acute and chronic administration of the melanocortin agonist, MTII. International Journal of Obesity 27 550-556.

Brakenhielm E, Cao R, Gao B, Angelin B, Cannon B, Parini P \& Cao Y $2004 a$ Angiogenesis inhibitor, TNP-470, prevents diet-induced and genetic obesity in mice. Circulation Research 94 1579-1588.

Brakenhielm E, Veitonmaki N, Cao R, Kihara S, Matsuzawa Y, Zhivotovsky B, Funahashi T \& Cao Y $2004 b$ Adiponectin-induced antiangiogenesis and antitumor activity involve caspase-mediated endothelial cell apoptosis. PNAS 101 2476-2481.

Bray GA \& Tartaglia LA 2000 Medicinal strategies in the treatment of obesity. Nature 404 672-677.

Broberger C, Johansen J, Johansson C, Schalling M \& Hokfelt T 1998 The neuropeptide Y/agouti gene-related protein (AGRP) brain circuitry in normal, anorectic, and monosodium glutamate-treated mice. PNAS 95 15043-15048.

Chun E, Han CK, Yoon JH, Sim TB, Kim Y-K \& Lee K-Y 2005 Novel inhibitors targeted to methionine aminopeptidase 2 (MetAP2) strongly inhibit the growth of cancers in xenografted nude model. International Journal of Cancer 114 124-130.

Crandall DL, Hausman GJ \& Kral JG 1997 A review of the microcirculation of adipose tissue: anatomic, metabolic, and angiogenic perspectives. Microcirculation 4 211-232.

Dhanabal M, Ramchandran R, Waterman MJF, Lu H, Knebelmann B, Segal M \& Sukhatme VP 1999 Endostatin induces endothelial cell apoptosis. Journal of Biological Chemistry 274 11721-11726.

Elmquist JK \& Marcus JN 2003 Rethinking the central causes of diabetes. Nature Medicine 9 645-647.

Fan W, Boston B, Kesterson R, Hruby V \& Cone R 1997 Role of melanocortinergic neurons in feeding and the agouti obesity syndrome. Nature 385 165-168.
Griffith EC, Su Z, Turk BE, Chen S, Chang YH, Wu Z, Biemann K \& Liu JO 1997 Methionine aminopeptidase (type 2) is the common target for angiogenesis inhibitors AGM-1470 and ovalicin. Chemistry and Biology 4 461-471.

Huszar D, Lynch C, Fairchild-Huntress V, Dunmore J, Fang Q, Berkemeier L, Gu W, Kersterson R, Boston B, Cone R et al. 1997 Targeted disruption of the melanocortin-4 receptor results in obesity in mice. Cell 88 131-141.

Ingber D, Fujita T, Kishimoto S, Sudo K, Kanamaru T, Brem H \& Folkman J 1990 Synthetic analogues of fumagillin that inhibit angiogenesis and suppress tumour growth. Nature 348 555-557.

Kopelman PG 2000 Obesity as a medical problem. Nature 404 635-643.

Lucas R, Holmgren L, Garcia I, Jimenez B, Mandriota SJ, Borlat F, Sim BKL, Wu Z, Grau GE, Shing Y et al. 1998 Multiple forms of angiostatin induce apoptosis in endothelial cells. Blood $\mathbf{9 2}$ 4730-4741.

Minokoshi Y, Kim Y-B, Peroni OD, Fryer LGD, Muller C, Carling D \& Kahn BB 2002 Leptin stimulates fatty-acid oxidation by activating AMP-activated protein kinase. Nature 415 339-343.

Mizuno T, Kleopoulos S, Bergen H, Roberts J, Priest C \& Mobbs C 1998 Hypothalamic pro-opiomelanocortin mRNA is reduced by fasting and in $o b / o b$ and $d b / d b$ mice, but is stimulated by leptin. Diabetes $\mathbf{4 7}$ 294-297.

Peruzzo B, Pastor FE, Blazquez JL, Schobitz K, Pelaez B, Amat P \& Rodriguez EM 2000 A second look at the barriers of the medial basal hypothalamus. Experimental Brain Research 132 10-26.

Rupnick MA, Panigrahy D, Zhang C-Y, Dallabrida SM, Lowell BB, Langer R \& Folkman MJ 2002 Adipose tissue mass can be regulated through the vasculature. PNAS 99 10730-10735.

Schwartz MW, Seeley RJ, Weigle DS, Burn P, Campfield LA \& Baskin DG 1997 Leptin increases hypothalamic proopiomelanocortin (POMC) mRNA expression in the rostral arcuate nucleus. Diabetes 46 294-297.

Schwartz MW, Woods SC, Porte D Jr, Seeley RJ \& Baskin DG 2000 Central nervous system control of food intake. Nature 404 661-671.

Seely R, Yagaloff K, Fisher S, Burn P, Thiele T, Van Dijk G, Baskin D \& Schwartz M 1997 Melanocortin receptors in leptin effects. Nature 390349.

Sharma HS \& Dey PK 1988 EEG changes following increased bloodbrain barrier permeability under long-term immobilization stress in young rats. Neuroscience Research 5 224-239.

Sin N, Meng L, Wang MQ, Wen JJ, Bornmann WG \& Crews CM 1997 The anti-antiogenic agent fumagillin covalently binds and inhibits the methionine aminopeptidase MetAP-2. PNAS 94 6099-6103.

Skultetyova I, Tokarev D \& Jezova D 1998 Stress-induced increase in blood-brain barrier permeability in control and monosodium glutamate-treated rats. Brain Research Bulletin 45 175-178.

Spurlock ME, Hahn KJ \& Miner JL 1996 Regulation of adipsin and body composition in the monosodium glutamate (MSG)-treated mouse. Physiology and Behavior 60 1217-1221.

Thiele T, Van Dijk G, Yagaloff K, Fisher S, Schwartz M, Burn P \& Seeley R 1998 Central infusion of melanocortin agonist MTII in rats: assessment of c-Fos expression and taste aversion. American Journal of Physiology 274 R248-R254.

Thornton JE, Cheung CC, Clifton DK \& Steiner RA 1997 Regulation of hypothalamic proopiomelanocortin mRNA by leptin in $o b / o b$ mice. Endocrinology 138 5063-5067.

Weigle DS 2003 Pharmacological therapy of obesity: past, present, and future. Journal of Clinical Endocrinology and Metabolism 88 2462-2469.

Woods SC, Seeley RJ, Porte D \& Schwartz MW 1998 Signals that regulate food intake and energy homeostasis. Science $\mathbf{2 8 0}$ $1378-1383$

Received in final form 2 January 2007

Accepted 11 February 2007

Made available online as an Accepted Preprint 14 February 2007 